\title{
Die Schweiz zahlt hohen Preis für nicht-übertragbare Krankheiten
}

\section{Carola A. Huber ${ }^{a}$, Simon Wieser ${ }^{b}$}

a Dr. phil., MPH, Versorgungsforscherin, Helsana-Gesundheitswissenschaften

b Prof. Dr. oec. publ., Leiter Winterthurer Institut für Gesundheitsökonomie, Zürcher Hochschule für Angewandte Wissenschaften

Ob Herz-Kreislauf-Erkrankungen, Krebs oder Atemwegserkrankungen: Solche sogenannten nicht-übertragbaren Krankheiten (non-communicable diseases, NCDs) stellen heutzutage die häufigste Todesursache von Frauen und Männern in der Schweiz dar. Ein interdisziplinäres Forschungsteam ermittelte erstmals die Krankheitskosten von nicht-übertragbaren Erkrankungen der Schweizer Bevölkerung.

Bislang war wenig darüber bekannt, wie sich die Gesundheitsausgaben in der Schweiz auf die Krankheiten verteilen. Transparenz schafft nun eine kürzlich im European Journal of Health Economics erschienene Studie, die erstmals systematisch und detailliert die Gesundheitskosten in der Schweiz aus dieser Perspektive beleuchtet [1]. Der Löwenanteil mit insgesamt 80 Prozent der Gesamtausgaben (51 von 65 Milliarden Franken im Jahr 2011) geht auf die sogenannten nichtübertragbaren Krankheiten zurück. Ganz unbekannt sind diese Zahlen nicht: Die Publikation basiert auf einem vom BAG in Auftrag gegebenen Bericht über die Kosten der nicht-übertragbaren Krankheiten, welcher 2014 von der Zürcher Hochschule für Angewandte Wis-

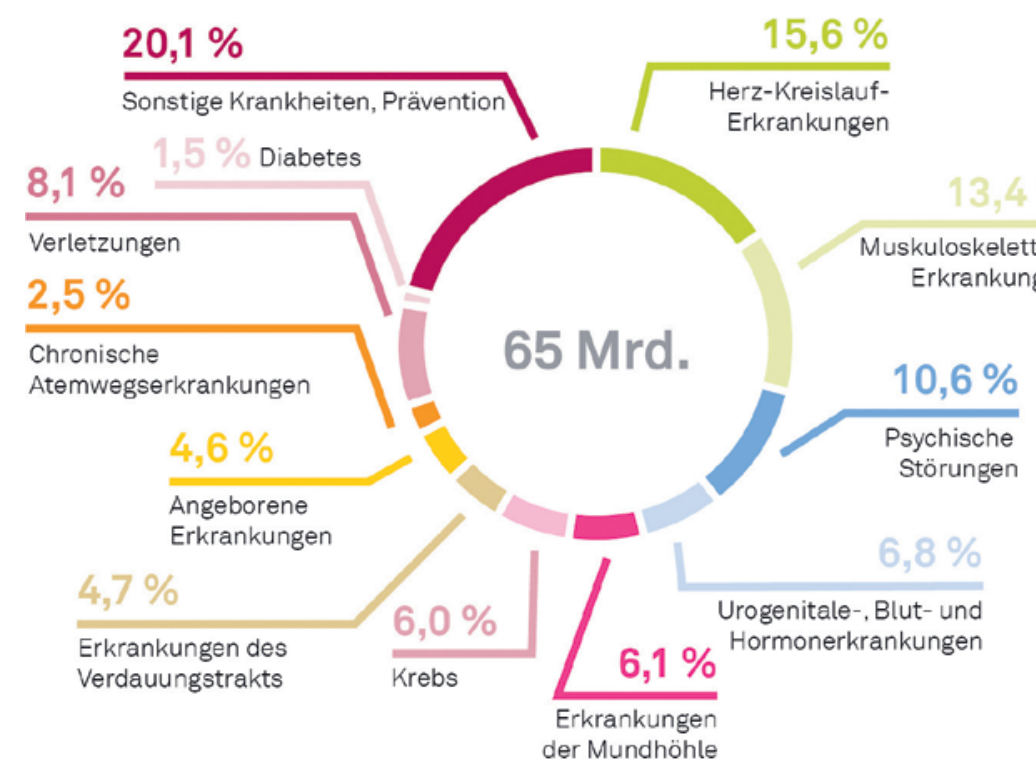

Abbildung 1: Schweizerische Gesundheitsausgaben nach Krankheitsgruppen. senschaften, der Universität Zürich, dem Forschungsinstitut Polynomics und der Helsana Krankenversicherung erstellt wurde.

Auf Platz 1 der teuersten Leiden rangieren die HerzKreislauf-Erkrankungen mit über 10 Milliarden Franken oder einem Anteil von 15,6 Prozent an den gesamten Gesundheitskosten (siehe Abb.1). Herz-Kreislauf-Erkrankungen stellen darüber hinaus die häufigste Todesursache von Männern und Frauen dar (über 20000 Todesfälle im Jahr) und sind zugleich der dritthäufigste Hospitalisierungsgrund in der Schweiz wie auch weltweit [2].

Mit 8,7 Milliarden Franken (13,4 Prozent) folgen an zweiter Stelle die muskuloskelettalen Erkrankungen wie Arthrose, Rheuma und Rückenschmerzen. Rang 3 belegen psychische Erkrankungen wie Depressionen mit fast 10,6 Prozent der Gesamtkosten (6,9 Milliarden Franken). Die häufig als Kostenfaktor diskutierten Krebserkrankungen belegen mit 6,0 Prozent der Gesamtkosten (3,9 Milliarden Franken) erst Rang 7, nach Verletzungen (5,2 Milliarden Franken bzw. 8,1 Prozent), urogenitalen, Blut- und Hormonerkrankungen (4,4 Milliarden bzw. 6,8 Prozent) und Erkrankungen der Mundhöhle (4 Milliarden bzw. 6,1 Prozent der Gesamtkosten).

\section{Die Studie misst die direkten Behandlungskosten}

Die Studie zeigt die direkten Kosten für die Behandlung einer Erkrankung. Kosten für die Behandlung anderer Krankheiten, welche durch eine Krankheit ausgelöst werden, sind in diesen nicht inbegriffen. Das Beispiel Diabetes veranschaulicht den Unterschied: 
Diabetes ist ein bedeutsamer Risikofaktor für HerzKreislauf-Erkrankungen. Die Kosten der durch Diabetes verursachten Herz-Kreislauf-Erkrankungen werden aber bei den Herz-Kreislauf-Erkrankungen und nicht bei Diabetes gezählt. Die durch Diabetes verursachten Behandlungskosten dürften de facto wesentlich höher ausfallen, als die 1,5 Prozent der Gesamtausgaben gemäss Studie. Für Osteoporose (eine muskuloskelettale Erkrankung) gilt eine ähnliche Überlegung: Obwohl Stürze von Osteoporose-Patienten häufig eine intensive stationäre Behandlung verlangen, werden die entsprechenden Gesundheitsausgaben bei den Verletzungen ausgewiesen, und nicht bei den muskuloskelettalen Erkrankungen.

\section{Rückenschmerzen - eine teure Volkskrankheit}

Muskuloskelettale Krankheiten belegen mit einem Anteil von 13 Prozent an den Gesamtausgaben Rang 2 der teuersten Krankheiten. Neben Arthrose und rheumatoider Arthritis sind hier vor allem Rückenschmerzen von Bedeutung. Das mag erstaunen, gelten die Rückentherapien doch gemeinhin nicht als teuer. Doch Rückenschmerzen sind sehr häufig. So litten im Jahr 2012 über 40 Prozent der erwachsenen Bevölkerung in den letzten vier Wochen daran (Schweizerische Gesundheitsbefragung [3]).

\section{Psychische Erkrankungen sind weit verbreitet}

Psychische Erkrankungen belegen Rang 3 der teuersten Krankheiten. Häufig ist von einer hohen Prävalenz die Rede. Wie hoch die Bezüge absolut sind, ist bis heute unbekannt. Helsana-Gesundheitswissenschaften arbeiten derzeit mit dem Psychologischen Institut der Universität Zürich an einer neuen Studie, um erstmals die Versorgungsrealität von Antidepressiva(AD)Verschreibungen in der Schweiz detailliert abzubilden. Eine erste Analyse/Erhebung ergab: 9 Prozent der Helsana-Grundversicherten im Jahr 2016 hatten mindestens eine AD-Verschreibung (110000 Bezüger aus total 1,2 Millionen Helsana-Grundversicherten). Die konservative Schätzung von Helsana geht von rund 730000 AD-Bezügern in der Schweiz aus (hochgerechnet auf Basis eines Helsana-Marktanteils von 15 Prozent).

Dass psychische Erkrankungen ein grosser Kostenfaktor sind, belegt der Helsana-Arzneimittelreport 2017 [4]. Obschon die absoluten Kosten für Psychostimulanzien in den letzten fünf Jahren leicht gesunken sind, stiegen in den Jahren 2013 bis 2016 die Bezüge von Psychostimulanzien in der Schweiz um mehr als 10 Prozent auf über 4,2 Millionen an.

Kommt hinzu: Über 60 Prozent der Kosten fallen im stationären Bereich an, also in den Kliniken (siehe

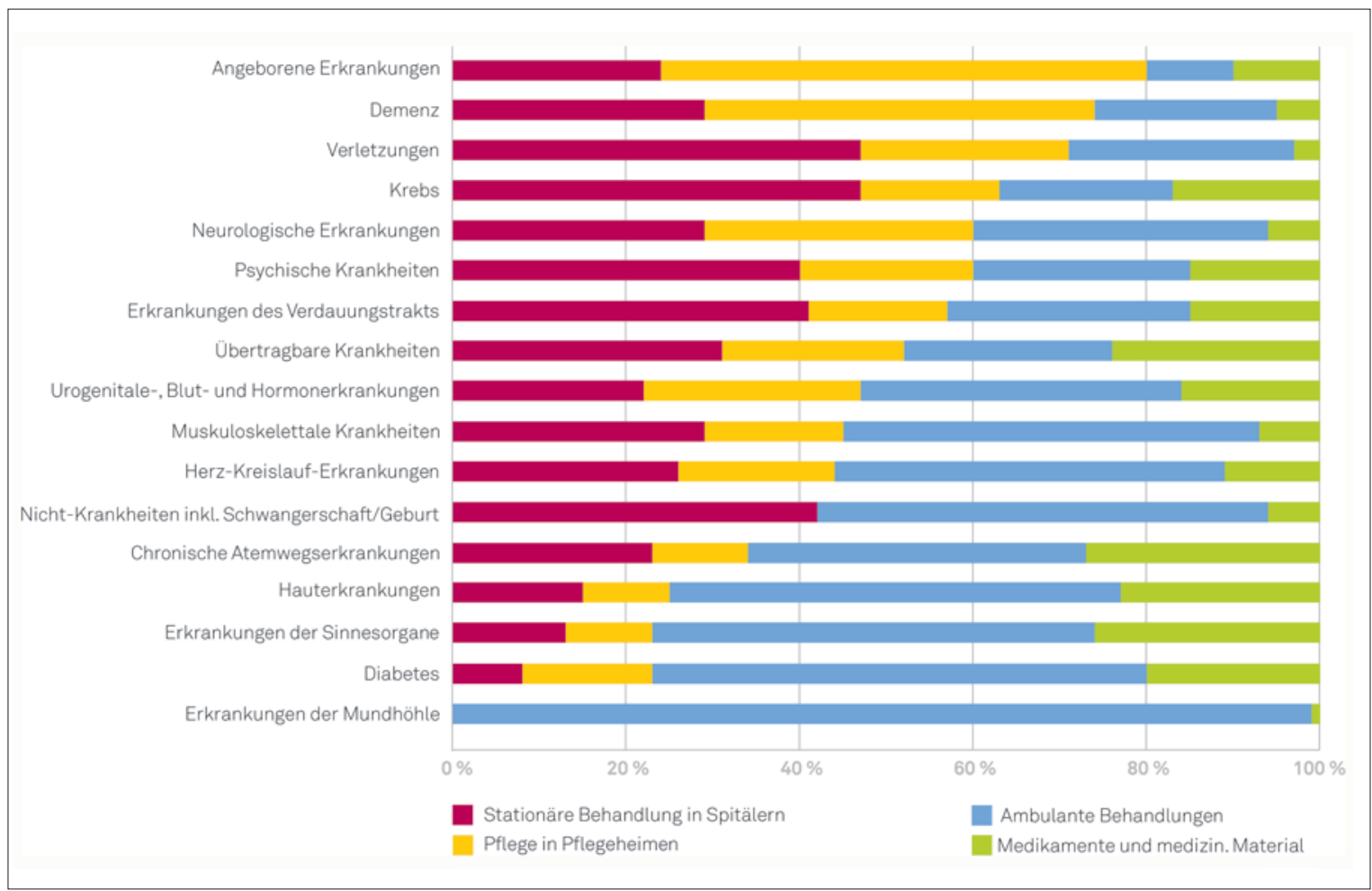

Abbildung 2: Anteile verschiedener Behandlungsarten an den Kosten der Krankheitsgruppen. 
Abb. 2). Hier gibt es Sparpotential, wenn die Therapien vermehrt ambulant erfolgen.

Überraschend ist: Demenz fällt nicht gross ins Gewicht. Obwohl die Krankheit immer wieder als Grund für die steigenden Gesundheitskosten angeführt wird, kosten Demenzerkrankungen lediglich 1,1 Milliarden Franken pro Jahr. Das macht weniger als zwei Prozent (1,7 Prozent) der gesamten Gesundheitskosten aus und liegt damit auf gleichem Rang wie die Erkrankungen der Sinnesorgane und Hauterkrankungen. Zum einen ist die Krankheit nicht so häufig, wie viele annehmen, zum anderen ist die Behandlung vieler Patienten für das Gesundheitswesen vergleichsweise günstig, da ein gewisser Anteil von ihren Angehörigen betreut wird.

\section{Krebs belegt den siebten Platz}

Während Krebs die zweithäufigste Todesursache ist, belegt die Krankheit mit 6 Prozent der Gesamtkosten den 7. Platz. Dieser tiefe Anteil ist zum einem auf eine oft relativ kurze Behandlungsdauer zurückzuführen: Die Zeit von der Diagnose bis zum Tod ist häufig vergleichsweise kurz - gerade bei schwer behandelbaren Formen wie dem Lungenkrebs. Zum anderen ist die Zahl der Erkrankten bei Krebs deutlich geringer als bei den Herz-Kreislauf-Erkrankungen oder den muskuloskelettalen Erkrankungen. Da der medizinische Fortschritt und manche neue (und teure) Medikamente die Lebenserwartung vieler Krebspatienten erhöhen, werden Krebserkrankungen jedoch immer mehr zu chronischen Erkrankungen [5].

\section{Integrierte Versorgung steigert Qualität und Effizienz}

Die Studie zeigt, dass chronische nicht-übertragbare Erkrankungen mit hoher Prävalenz die schweizerischen Gesundheitskosten dominieren. Häufiger tödlich verlaufende Krankheiten, wie viele Krebsarten, haben hingegen einen vergleichsweise geringeren Anteil an den Gesamtkosten.

Angesichts dieser Erkenntnisse stellt sich die Frage, wie die hiesige Versorgung effizienter gestaltet werden kann, um das Kostenwachstum zu bremsen. Gerade für chronische Leiden wie Herz-Kreislauf-Erkrankungen, Diabetes und Rückenschmerzen kann die integrierte Versorgung (Managed Care) einen grossen Beitrag zu einer qualitativ besseren und effizienteren Versorgung leisten [6].

\section{Literatur}

1 Wieser S, Riguzzi M, Pletscher M, Huber CA, Telser H, Schwenkglenks $M$. How much does the treatment of each major disease cost? A decomposition of Swiss National Health Accounts. Eur J Health Econ. 2018; https://doi.org/10.1007/s10198-018-0963-5.

2 Bundesamt für Gesundheit BAG. https://www.bag.admin.ch/bag/ de/home/themen/mensch-gesundheit/nichtuebertragbare-krankheiten/herz-kreislauf-erkrankungen.html

3 Schweizerische Gesundheitsbefragung 2012, Bundesamt für Statistik (2013). https://www.bfs.admin.ch/bfs/de/home/statistiken/ gesundheit/erhebungen/sgb.html

4 Helsana. Arzneimittelreport für die Schweiz 2017; S. 191. https:// www.helsana.ch/docs/arzneimittelreport-2017.pdf

5 https://www.krebsgesellschaft.de/onko-internetportal/basis-informationen-krebs/basis-informationen-krebs-allgemeine-informationen/krebs-bald-eine-chronische-erkr.html

6 Huber CA, Reich O, Früh M, Rosemann T. Effects of Integrated Care on Disease-Related Hospitalisation and Healthcare Costs in Patients with Diabetes, Cardiovascular Diseases and Respiratory Illnesses: A Propensity-Matched Cohort Study in Switzerland. Int J Integrated Care. 2016;16(1):11. 